Article

\title{
Characterization of Dermal Fibroblasts as a Cell Source for Pediatric Tissue Engineered Heart Valves
}

\author{
Monica M. Fahrenholtz ${ }^{1}$, Huiwen Liu ${ }^{1}$, Debra L. Kearney ${ }^{2}$, Lalita Wadhwa ${ }^{3}$, \\ Charles D. Fraser, Jr. ${ }^{3}$ and K. Jane Grande-Allen ${ }^{1, *}$
}

1 Department of Bioengineering, Rice University, 6100 Main Street MS-142, Houston, TX 77005, USA; E-Mails: mmf4@rice.edu (M.M.F.); h125@rice.edu (H.L.)

2 Department of Pathology, Texas Children's Hospital, 6621 Fannin Street, Houston, TX 77030, USA; E-Mail: dlkearne@texaschildrens.org

3 Department of Congenital Heart Surgery, Texas Children's Hospital, 6621 Fannin Street, Houston, TX 77030, USA; E-Mails: 1xwadhwa@texaschildrens.org (L.W.); cdfraser@texaschildrens.org (C.D.F.)

* Author to whom correspondence should be addressed; E-Mail: grande@rice.edu; Tel.: +1-713-348-3704; Fax: +1-713-348-5877.

Received: 17 June 2014; in revised form: 29 July 2014 / Accepted: 20 August 2014 / Published: 26 August 2014

\begin{abstract}
There is continued debate regarding the appropriate cell type to replace valvular interstitial cells (VICs) in tissue engineered heart valves (TEHVs), particularly for pediatric patients. In this work, neonatal human dermal fibroblasts (nhDFFs) were compared to human pediatric VICs (hpVICs), based on their phenotypic and gene expression characteristics when cultured on collagen type I, fibronectin, fibrin, and tissue culture polystyrene (TCP) substrates. Similar confluency was achieved over the culture period on collagen and fibronectin between both cell types, although nhDFFs tended to reach lower confluence on collagen than on any other substrate. Morphologically, hpVICs tended to spread and form multiple extensions, while nhDFFs remained homogenously spindle-shaped on all substrates. PCR results indicated that fibroblasts did not differ significantly from VICs in gene expression when cultured on fibrin, whereas on collagen type I and fibronectin they showed increased $\alpha$-SMA, xylosyltransferase I, and collagen type I expression $(p<0.05)$. However, protein expression of these targets, analyzed by immunocytochemistry and Western blotting, was not significantly different between cell types. These results suggest
\end{abstract}


that nhDFFs express similar matrix production and remodeling genes as hpVICs, and the choice of substrate for TEHV construction can affect the growth and expression profile of nhDFFs as compared to native hpVICs.

Keywords: fibroblasts; pediatric; valvular interstitial cell; VIC; TEHV; extracellular matrix; substrate

\section{Introduction}

Congenital heart defects affect nearly $1 \%$ of all live births and are the leading cause of death in infants with birth defects in the United States [1,2]. Many of these defects, including bicuspid aortic valve and tetralogy of Fallot, significantly affect the function of the heart valves. This necessitates valve replacement in patients from newborn to 18 years of age, often with additional replacement surgeries in adulthood. Tissue engineered heart valves (TEHVs) are often considered the ideal valve replacement for pediatric patients with congenital heart defects primarily because they can serve as a scaffold for seeded cells, which can then remodel the valve and allow it to grow with the patient over time, alleviating the need for additional replacement surgeries [3,4]. Furthermore, TEHVs could be engineered to encourage the re-growth and maintenance of an endothelial lining, naturally protecting the valve from inflammation and thrombosis [5-8]. No other currently available replacement option offers these capabilities. However, there is continued debate about what type of cell would be appropriate to seed in these tissue engineered valves to enable appropriate remodeling, in addition to a limited knowledge of the characteristics of valve cells from the pediatric population.

In normal valves, valvular interstitial cells (VICs) are responsible for valve remodeling in response to mechanical forces and maintenance of valve extracellular matrix [9,10]. VICs are observed to be a highly heterogeneous population with two primary phenotypes: quiescent (qVICs) and activated (aVICs). qVICs are most commonly found in fully-developed adult valve tissue, where they maintain valve phenotype by replacing old or damaged matrix [11,12]. These cells are characterized by slow turnover and a lack of $\alpha$-smooth muscle actin ( $\alpha$-SMA) expression $[11,13,14]$. By contrast, aVICs are characterized by expression of $\alpha$-smooth muscle actin, much like myofibroblasts, and actively proliferate and produce matrix, usually in response to disease or other abnormal conditions $[11,12]$. Due to the rapid growth and remodeling in fetal tissues, aVICs are the most common fetal VIC phenotype. Some remodeling continues after birth and through early childhood in children as old as six to seven years, but eventually most cells will switch to the quiescent phenotype seen predominantly in adult tissues $[12,13,15]$. Because of this progression of remodeling towards a normal adult valve structure, pediatric VICs differ significantly from adult VICs, maintaining higher cell density, increased expression of matrix remodeling proteins, and contributing to increasing collagen alignment and elastin deposition during development [13]. This distinction has important implications for choosing a suitable cell type to replace VICs in TEHVs for pediatric applications, necessitating the direct comparison of pediatric VICs to surrogate cell types for pediatric-specific applications. Unfortunately, most studies to date that have characterized VICs and compared their behavior to other cell types have been carried out using adult human or animal VICs [11,16-21]. Therefore, there is a 
need for direct comparison of alternative cell types to pediatric VICs in order to choose an appropriate surrogate for developing pediatric-specific TEHVs.

One cell type that has garnered attention as a potential VIC replacement cell type is the dermal fibroblast [18,22-26]. These cells are straightforward to obtain via biopsy and to culture in vitro, making them attractive for autograft applications. Many reports have cited their similarity to VICs, because of their expression of $\alpha$-SMA as well as prolyl-4-hydroxylase and other collagen producing and remodeling molecules [18,19,24,25]. Currently, limited information is known about how the matrix remodeling and production capabilities of fibroblasts compare specifically to pediatric VICs, and the general behavior of pediatric VICs. Many studies have focused on media supplements to modulate the behavior of fibroblasts, but have not investigated the effects of different extracellular matrix molecules, which are much easier to covalently incorporate into a TEHV construct [16,18,24,27]. Clearly, further study is needed to determine whether dermal fibroblasts can recapitulate the major functions of VICs, whether they are suitable for pediatric TEHV applications, and how matrix molecules may influence cell behavior.

In this work, we investigated the effect of substrate on the behavior of neonatal human dermal foreskin fibroblasts (nhDFFs) and human pediatric VICs (hpVICs) to determine by direct comparison whether nhDFFs are an appropriate hpVIC surrogate for pediatric-specific TEHVs. In particular, cells were cultured on collagen type I and fibronectin, extracellular matrix molecules that are commonly found in the native valve [28-30], and on fibrin, which has been used frequently as a TEHV construction material $[18,31,32]$. Finally, cells were also grown on tissue culture polystyrene (TCP) for a typical in vitro culture control. A thorough comparison of the functional characteristics of both cell types was performed by investigating important targets related to matrix remodeling and production. Matrix metalloproteinase I and tissue inhibitor of matrix metalloproteinase I were chosen to investigate matrix remodeling $[12,33]$. Collagen type I was also studied, as it is the most common matrix protein in the valve, providing tensile strength in the tissue, as well as prolyl-4-hydroxylase, which is an important enzyme in collagen synthesis [29]. Additionally, $\alpha$-SMA was chosen as an indicator of activation for both VICs and fibroblasts. Finally, xylosyltransferase I was investigated because of its essential role in glycosaminoglycan (GAG) chain production [34]. GAGs are a common and essential matrix component of the valve, and contribute to the flexibility and compressive resistance of the valve structure [29]. Previous work to find VIC surrogates have focused on adult VICs compared to various cell types, thus, hpVICs are understudied in the literature to date. This work will demonstrate whether fibroblasts can recapitulate pediatric VIC behavior and function and provide guidance towards engineering a pediatric TEHV matrix to encourage hpVIC-like function by fibroblasts.

\section{Experimental Section}

\subsection{Materials}

Collagen type I and fibronectin coated culture vessels were purchased from BD Biosciences (San Jose, CA, USA). Fibrin coated plates were produced by adsorption of $100 \mu \mathrm{g} / \mathrm{mL}$ fibrinogen (Sigma-Aldrich, St. Louis, MO, USA) onto a TCP vessel for $1.5 \mathrm{~h}$ at $37^{\circ} \mathrm{C}$, followed by clotting with $0.04 \mathrm{mg} / \mathrm{mL}$ thrombin (82.5 U/mg, Fisher Scientific, Pittsburg, PA, USA) for $10 \mathrm{~min}$ at $37{ }^{\circ} \mathrm{C}$ [35]. 


\subsection{Cell Isolation and Culture}

hpVICs were isolated from fresh valve tissue (mean patient age 10 years) harvested during valve repair surgery at Texas Children's Hospital in accordance with IRB protocols 11-176E approved on 22 April 2011 at Rice University and H-18511 approved on 21 August 2011 by Baylor College of Medicine and its affiliated hospitals. Patient characteristics are summarized in Table 1. For this study, we were unable to obtain fresh valves from autopsy, due to the rarity of such samples from the pediatric population, and the complications in obtaining such samples within $24 \mathrm{~h}$ for maximum cell viability. Isolation of hpVICs was performed as described previously [36]. Briefly, leaflet samples were digested in collagenase II and brushed vigorously with a cotton swab to remove any remaining endothelial cells. A second digestion in collagenase III was performed to release the hpVICs from the extracellular matrix. Collected cells were then plated on a T-75 TCP flask. nhDFFs were purchased from ATCC (PCS-201-010, ATCC, Manassas, VA, USA). Both cell types were cultured in 1:1 DMEM/F12 (Corning, Manassas, VA, USA/HyClone, Logan, UT, USA) with 10\% bovine growth serum (HyClone) and 1\% antibiotic-antimicotic solution (Corning) on TCP, as described previously [37]. Media was supplemented with $100 \mu \mathrm{g} / \mathrm{mL}$ tranexamic acid to prevent the immediate digestion of the fibrin coating by the nhDFFs. At passage 2, cells were seeded at $10^{4}$ cells $/ \mathrm{cm}^{2}$ on 6 -well plates and in 8 -well chamber slides coated with rat-tail collagen type I, fibronectin, fibrin, or no coating (TCP only) [37,38]. Two or three biological replicates for each patient were analyzed in each assay, resulting in six to eight total samples. Cells were cultured for seven days at $37{ }^{\circ} \mathrm{C}$ and $5 \% \mathrm{CO}_{2}$ to allow the cells time to respond to the substrates and come to a "steady-state" behavior. Cells cultured in 6-well plates were used for RNA and protein isolation, while cells cultured in 8-well chamber slides were analyzed by immunocytochemistry.

Table 1. Patient information.

\begin{tabular}{ccccc}
\hline Patient No. & Age (years) & Sex & Valve & Diagnosis \\
\hline 1 & 13 & F & Aortic & Aortic regurgitation \\
\hline 2 & 5 & M & Aortic & Aortic stenosis \\
\hline 3 & 12 & M & Aortic & $\begin{array}{c}\text { Truncus arteriosus, DiGeorge Syndrome, VSD, } \\
\text { Aortic Regurgitation }\end{array}$ \\
\hline
\end{tabular}

\subsection{Phenotypic Assays}

To analyze differences in growth and proliferation, cell area was determined by tracing the outline of cells stained by phalloidin, a cytoskeletal marker, and DAPI was used to visualize the cell nuclei and perform a cell count for each experimental condition, all in ImageJ (NIH, Bethesda, MD, USA). Using the cell area and cell density determined from image analysis, the percent coverage of a $1 \mathrm{~cm}^{2}$ culture area was calculated.

For immunocytochemistry, hpVICs and nhDFFs cultured in 8-well chamber slides were fixed with 4\% paraformaldehyde in PBS for $10 \mathrm{~min}$ at room temperature (RT), then fluorescently stained to confirm the expression of $\alpha$-SMA and P4H proteins, since phenotypic markers have been commonly used in the literature to identify VICs and fibroblasts. First, a permeabilization step was performed by incubation with $2 \%$ Triton-X in PBS for 15 min at RT, then the cells were blocked with $2 \%$ BSA in 
PBS with $\mathrm{Ca}^{++}$and $\mathrm{Mg}^{++}$. Next, primary antibodies at a dilution of 1:50 for $\alpha$-SMA (ab 7817, Abcam, Cambridge, MA, USA) and 1:100 for P4H (bs-5090R, Bioss, Woburn, MA, USA) were allowed to bind for $1 \mathrm{~h}$ at $37^{\circ} \mathrm{C}$. After rinsing in PBS with $\mathrm{Ca}^{++}$and $\mathrm{Mg}^{++}$, the primary antibodies were detected with AlexaFluor-594-conjugated, anti-mouse secondary antibodies (Life Technologies) at a dilution of 1:200 for $1 \mathrm{~h}$ at RT. Cells were counterstained with DAPI for $5 \mathrm{~min}$ at RT to show the cell nuclei. Negative controls were performed by adding the primary or secondary antibody alone to a cell sample. PBS was used to wash the wells, then more PBS was added to each slide well, and slides were imaged using a Nikon TE300 inverted fluorescent microscope (Nikon, Melville, NY, USA). Images were processed using Image $(\mathrm{NIH})$ and false-colored to improve visualization.

\subsection{RNA and Protein Isolation}

Total RNA was isolated from nhDFFs and hpVICs with TRIzol reagent (Ambion, Grand Island, NY, USA) according to the manufacturer's protocol and suspended in RNase-free water. RNA concentration was measured using a Nanodrop spectrophotometer. Protein was precipitated from the organic phase of the TRIzol sample using an ethanol extraction protocol as described by the manufacturer, then resuspended in $2 \%$ SDS. The concentration of protein in each sample was measured using the DC Protein Assay (Bio-Rad, Hercules, CA, USA).

\subsection{Quantitative PCR}

One microgram of RNA was converted to cDNA with a BluePrint 1st Strand cDNA Synthesis Kit (Takara, Mountain View, CA, USA). To analyze relative gene expression, qPCR was performed to amplify the following genes of interest (Table 2): collagen I (COL1), $\alpha$-SMA, tissue inhibitor of metalloproteinase I (TIMP1), matrix metalloproteinase I (MMP1), xylosyltransferase I (XYLT1), and prolyl-4-hydroxylase (P4H). Glyceraldehyde 3-phosphate dehydrogenase (GAPDH) was used as a housekeeping gene. All primers were designed using Primer Designer software (Premier Biosoft, Palo Alto, CA, USA) and custom-made oligos were ordered from Integrated DNA Technologies (Coralville, IA, USA). Sequences are given in Table 2.

Table 2. Sense and antisense primers used for qPCR.

\begin{tabular}{cll}
\hline Target Gene & \multicolumn{1}{c}{ Sense Primer } & \multicolumn{1}{c}{ Anti-Sense Primer } \\
\hline COL1 & AATCACCTGCGTACAGAACGG & TCGTCACAGATCACGTCATCG \\
$\alpha$-SMA & CGTGTTGCCCCTGAAGAGCAT & ACCGCCTGGATAGCCACATACA \\
TIMP1 & CATCCTGTTGTTGCTTGGCT & ACGCTGGTATAAGGTGGTCTG \\
MMP1 & GGCTGGGAAGCCATCACTTACC & ACACCACACCCCAGAACAGCA \\
XYLT1 & TTCCTCCAACTTGCTTCT & ATGCTGTCTTCTCTGATGT \\
P4H & TCTAACGGCTGAGGACTGCTT & CTCGCCTTCATCCAGTTGCCT \\
GAPDH & CCACTCCTCCACCTTTGAC & ACCCTGTTGCTGTAGCCA \\
\hline
\end{tabular}

The Advantage qPCR premix kit (Clontech, Mountain View, CA, USA) was used for gene amplification and measurement with SYBR green. Fluorescent intensity was converted to a relative expression level using a modified $\Delta \Delta \mathrm{Ct}$ method that accounts for primer efficiency and normalizes to GAPDH [39]. To investigate differences between the two cell types on each substrate, fibroblasts on 
each substrate were normalized to hpVICs on the same substrate and a Student's $t$-test assuming unequal variance was used to compare gene expression of nhDFFs and hpVICs. Samples of each cell type were also normalized separately to either hpVICs or nhDFFs on TCP to determine if there was any effect of substrate on the gene expression profile for each cell type alone.

\subsection{Western Blotting}

For Western blotting, $10 \mu \mathrm{g}$ of protein was diluted in $7.5 \mu \mathrm{L}$ deionized water and mixed with $5 \mu \mathrm{L}$ NuPage ${ }^{\circledR}$ LDS sample buffer (Life Technologies), then samples were incubated at $70{ }^{\circ} \mathrm{C}$ in a heat block for 10 min. Samples were next loaded in a pre-cast 4\%-10\% Bis-Tris gel with SDS-MOPS running buffer (Life Technologies) and electrophoresed at $200 \mathrm{~V}$ for approximately $70 \mathrm{~min}$. Molecular weight was assessed by comparison with a pre-stained standard (Novex). Protein was transferred to a nitrocellulose membrane using a semi-dry transfer chamber (Bio-Rad) with Bjerrum and Schafer-Nielsen transfer buffer. Detection was performed by blocking the membrane in $0.1 \%$ Tween-20 in TBS with $2 \%$ nonfat dry milk and $2 \%$ bovine serum albumin overnight at $4{ }^{\circ} \mathrm{C}$. Then, the membrane was treated with primary antibodies for collagen type I (ab34710, Abcam), MMP1 (ADI-905-472, Enzo Life Sciences, Farmingdale, NY, USA), and TIMP1 (ab1827, Abcam) to show production of the primary ECM and ECM remodeling proteins with $\beta$-tubulin (ab6046, Abcam) as a loading control in $0.1 \%$ Tween-20 in TBS (dilution 1:2000-5000) for $2 \mathrm{~h}$ at RT. Next, the membrane was washed four times in TBS-Tween and treated with horseradish peroxidase-conjugated secondary antibodies (ab97051 and ab97023, Abcam) at a dilution of 1:5000-10,000 in TBS-Tween for $1 \mathrm{~h}$ at room temperature. Proteins were detected with ECL Prime Western blotting detection reagent (Amersham, Pittsburgh, PA, USA) and imaged with an LAS4000 imaging system (Thermo). Quantification of the resulting images was carried out in ImageJ, and samples were normalized to the data from nhDFFs grown on tissue culture plastic and to the loading control, $\beta$-tubulin.

\subsection{Statistical Analysis}

Cells isolated from three patients were used in the study to control for patient-to-patient variability in hpVIC gene and protein expression. Experiments were performed in duplicate or triplicate for each patient, and data were pooled across all replicates for analysis to investigate the differences between hpVICs and nhDFFs as a population ( $n=8$, unless otherwise noted). Equal replicates were performed using nhDFFs in parallel with the hpVICs. Unless otherwise described above, statistical analysis was performed by conducting a one-way ANOVA with a post hoc Tukey's test to assess differences between groups, and significance was accepted at $p<0.05$.

\section{Results and Discussion}

\subsection{Comparison of nhDFF and hpVIC Morphology}

Morphological differences between nhDFFs and hpVICs were observed by day five of the culture period (Figure 1). Fibroblasts exhibited a uniform spindle-like morphology and quickly reached high confluence in culture, as expected. By contrast, the hpVIC population was more heterogeneous, i.e., some cells appeared more spindle-like, whereas many others spread and formed multiple extensions. 
In fact, the average surface area of hpVICs was significantly larger than nhDFFs $\left(697 \pm 125 \mu \mathrm{m}^{2} v s\right.$. $338 \pm 73 \mu^{2}$, respectively, $p<0.0001$ ). Additionally, hpVICs tended to form a loose network at confluence, in contrast to the close-packed layer of the nhDFFs, similar to that observed for adult qVICs in culture [12].

Figure 1. Representative light microscopy images of nhDFFs (A-D) and hpVICs $(\mathbf{E}-\mathbf{H})$ after five days of culture on various substrates: TCP $(\mathbf{A}, \mathbf{E})$, fibronectin $(\mathbf{B}, \mathbf{F})$, fibrin $(\mathbf{C}, \mathbf{G})$, and collagen type I (D,H). hpVICs appear to spread more on all substrates and form less dense colonies, while fibroblasts appear to reach a higher degree of confluence with homogeneous, spindle-shaped morphology. Scale bar $=100 \mu \mathrm{m}$.

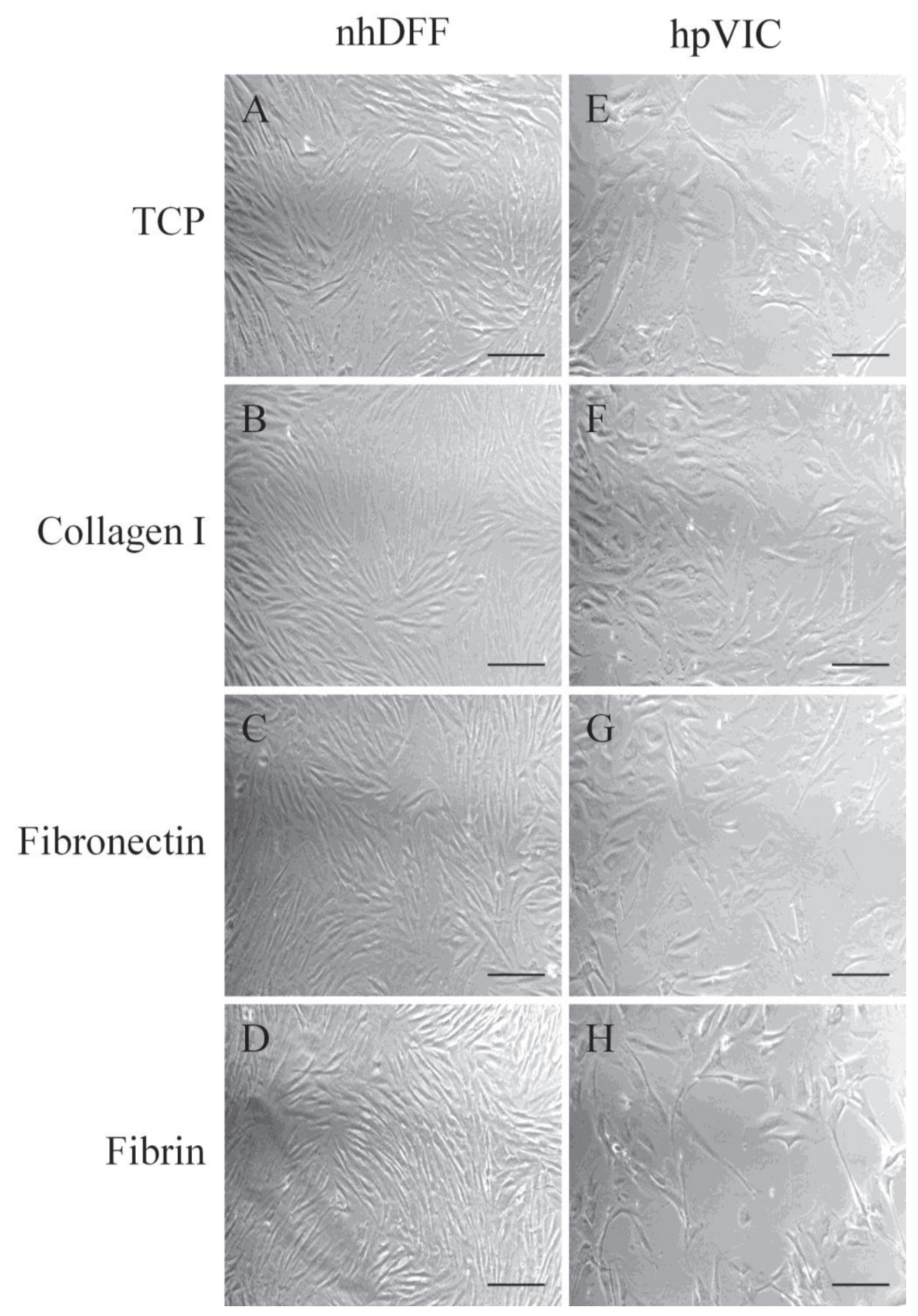


Figure 2. Average percent area coverage of substrates at seven days of culture. Area coverage was significantly different between nhDFFs and hpVICs on fibrin and TCP. nhDFFs showed a trend towards decreasing confluency on collagen as compared to fibronectin and TCP $(0.05<p<0.1)$. Data is given as mean $\pm \mathrm{SE}$, with $* p<0.05$.

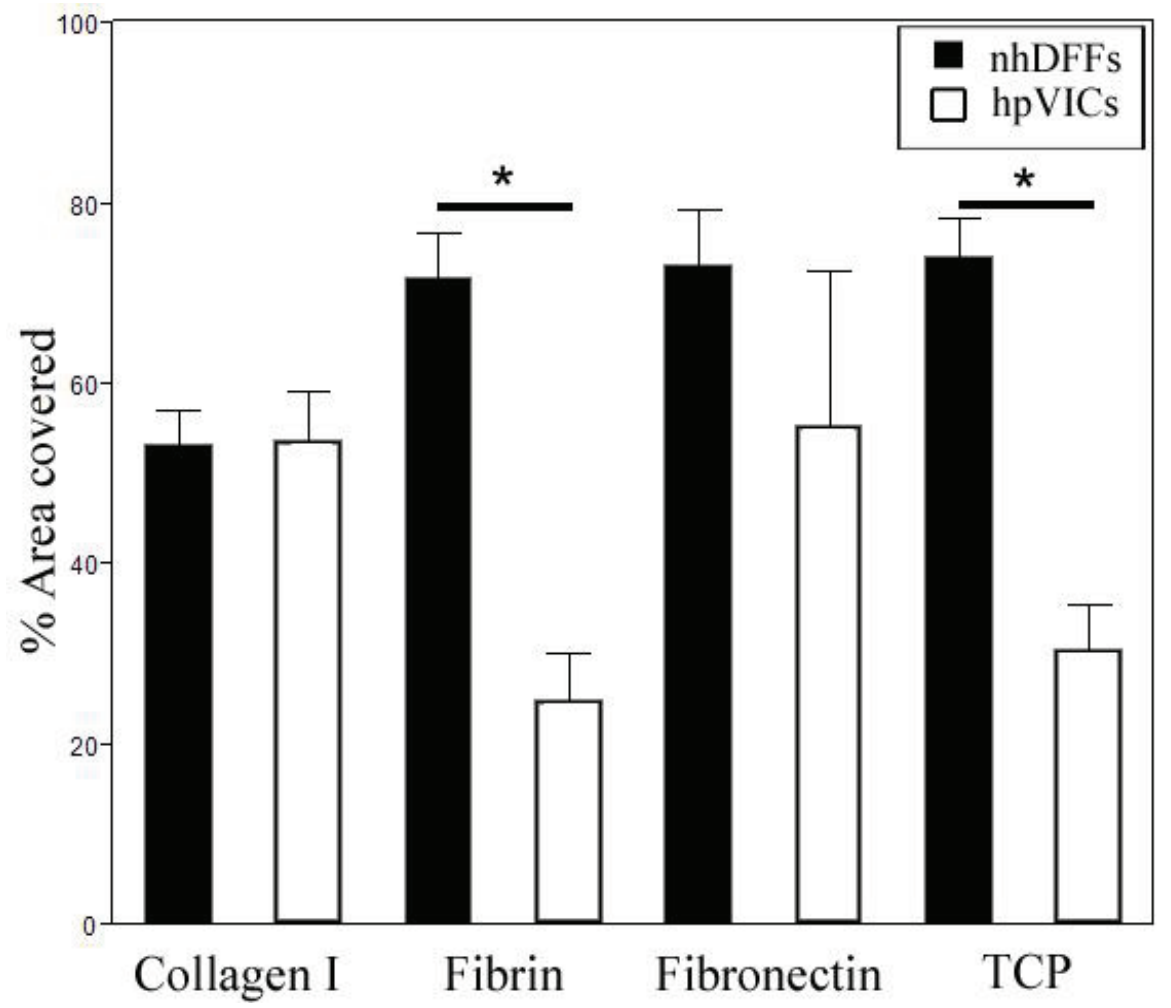

Analysis of the percent area covered by nhDFFs and hpVICs revealed interesting differences in behavior on certain matrix substrates (Figure 2). On native valve matrix components, collagen type I and fibronectin, nhDFFs and hpVICs reached similar levels of confluence. Culture on fibrin and TCP, however, resulted in a significant decrease in the surface coverage by hpVICs as compared to nhDFFs $(p<0.01)$. In fact, nhDFFs appeared to grow best on these substrates, as well as fibronectin. Collagen type I, by contrast, tended to promote less confluent fibroblast cultures than either fibronectin or TCP, the two substrates with the highest percent coverage by nhDFFs $(0.05<p<0.1)$. It is important to note that while apoptosis was not specifically studied, significant decreases in cell number were not observed during the culture period. This is consistent with the low expression of apoptotic markers observed previously in pediatric valve sections [13]. Based on these results, it seems that components of the native valve matrix better supports the growth of hVICs, likely due to the more natural biochemical environment experienced on these substrates. The difference between the growth of nhDFFs and $\mathrm{hpVICs}$ on the fibrin substrate could be due to their differential expression of integrins, which mediate cell attachment to various matrices, including fibrin and fibronectin. Fibroblasts significantly express $\alpha_{v} \beta_{3}$ integrin in order to migrate in wound environments [40-43], thus, fibrin would support the growth and proliferation of nhDFFs. The integrin expression profile of hpVICs has not been extensively studied, however, since fibrin is not part of the native valve matrix, nor is it present in disease states, hpVICs may not significantly express $\alpha_{\mathrm{v}} \beta_{3}$ integrin. Furthermore, results from previous studies may suggest that these cells do not express high levels of integrins in general because of their distinct 
interactions with matrix, particularly in disease states, as compared to adult VICs. For example, A report by Fisher, et al. [44] found that strain magnification was able to encourage calcific nodule formation by adult porcine VICs, and another study by Gu and Masters [35] showed the essential role of integrins in the calcification process of adult porcine VICs. These results, taken together with the tendency of pediatric VICs not to form calcific nodules [45] seems to suggest that they lack some of the integrin-based feedback mechanisms that enhance calcification in adult VICs and lead to growth and proliferation of fibroblasts. Similar to nhDFFs cultured on fibrin, TCP and fibronectin likely promoted higher growth in nhDFFs due to the cells' role in wound healing. Collagen, as mentioned above, seemed to reduce the highly proliferative, "wound healing"-like response of fibroblasts, resulting in area coverage that was very similar between both cell types [46]. This is most likely because this matrix component is commonly found in the native environment for both VICs and fibroblasts, promoting more "natural" cell growth.

Figure 3. Fluorescence imaging after seven days in culture showed highly heterogeneous staining for $\alpha$-SMA (red) with a counter-stain for nuclei (blue, DAPI) in both nhDFF and hpVIC cultures on all substrates: TCP, collagen type I, fibronectin, and fibrin (A). In all samples, $\alpha$-SMA intensity ranged from high intensity to very low or no signal throughout the culture, but no significant differences were observed in area positive for $\alpha$-SMA (B). Scale bars $=100 \mu \mathrm{m}$, and data is expressed as mean \pm standard error.

A

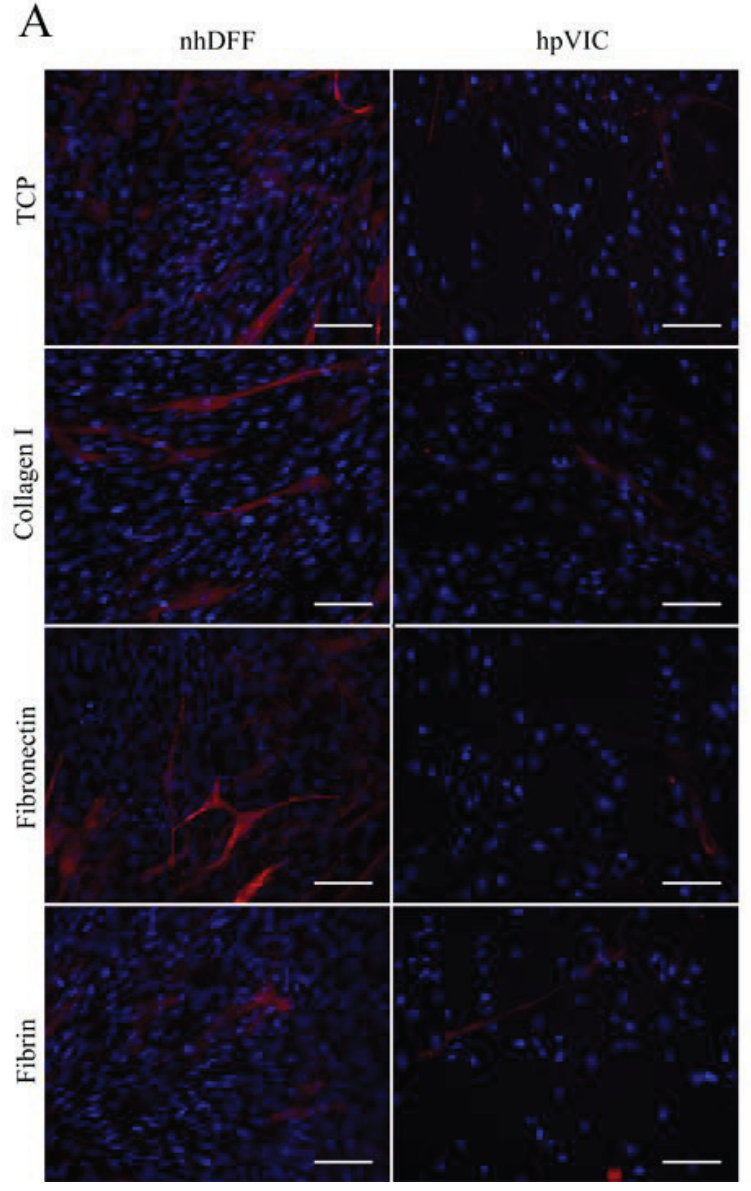

B

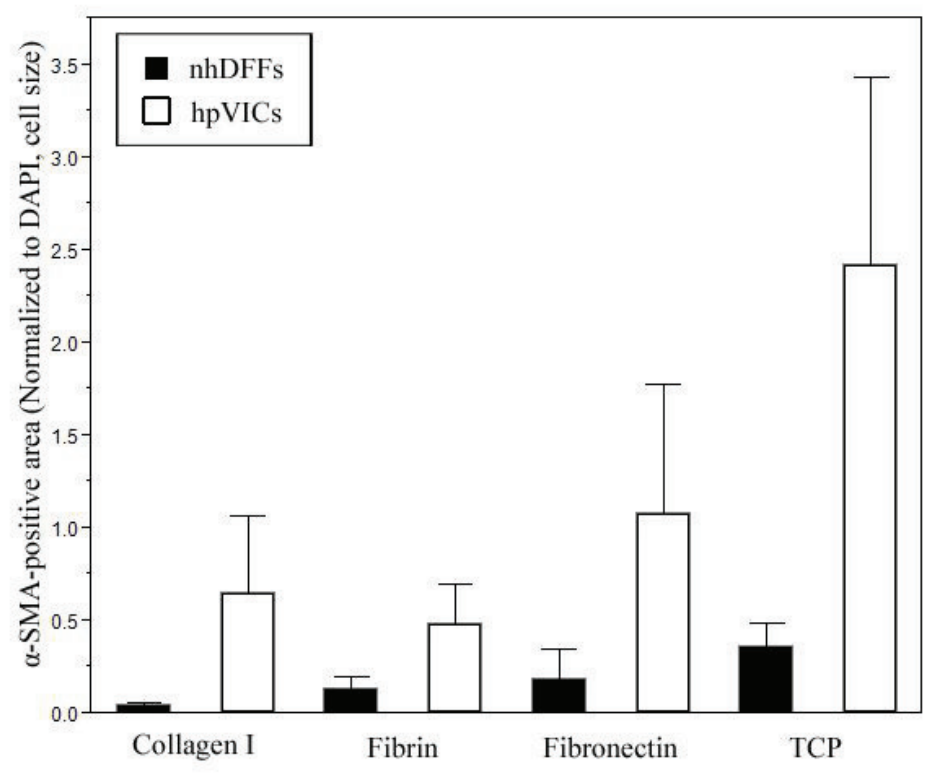




\subsection{Immunofluorescence}

Immunofluorescent imaging of cells showed low-level, homogeneous expression of prolyl-4hydroxylase by both nhDFFs and hpVICs, with no significant differences between substrate types (Supplementary Material, Figure S1), as expected from previous studies [19,33,47]. Staining for $\alpha$-SMA expression revealed a very heterogenous expression profile in both cell types. As shown in Figure 3A, cells in all conditions qualitatively exhibited a wide range of expression for $\alpha$-SMA, from no staining to strong staining, as has been previously reported for normal adult VIC populations in vitro [19]. Normalized area positive for $\alpha$-SMA was not significantly different in any condition; however, it was noted that there appeared to be greater variability in the expression of $\alpha$-SMA for hpVIC cultures than nhDFFs (Figure 3B). There was no significant staining observed in either control condition. While significant differences in confluence over the culture period were observed, there was not a marked change in $\alpha$-SMA expression in any condition that would indicate significant activation of either cell type.

\section{3. $q P C R$}

To determine the differences in the gene expression response between the two cell types on each substrate, fibroblast expression of gene targets was normalized to expression by hpVICs on matched substrates. On both collagen type I and fibronectin surfaces, responses by nhDFFs and hpVICs were significantly different for multiple gene targets. As shown in Figure 4, gene expression of $\alpha$-SMA and xylosyltransferase I were significantly greater in nhDFFs. Additionally, collagen type I expression was upregulated on fibronectin. No significant differences in gene expression between the two cell types when grown on fibrin, but cultures on TCP showed upregulation of collagen type I and xylosyltransferase I in nhDFFs. In an alternative analysis to test the effects of substrate on a given cell type, qPCR results were normalized to data from cells cultured on tissue culture plastic. However, this manner of normalization did not reveal any significant effect of culture substrate on the gene expression profile for either cell type.

These results imply that the TCP surface was less amenable to hpVIC growth, as shown in Figure 2, because hpVICs do not express matrix production-related genes as strongly as nhDFFs; therefore, they may have fewer signals from extracellular matrix proteins to encourage their growth. In general, on the above substrates, nhDFFs have a more activated, matrix-producing gene profile, especially on collagen type I and fibronectin. In addition, the upregulation of COL1 on fibronectin and TCP indicates a high potential for matrix production, which would be expected in the context of a wound response like that of nhDFFs naturally on fibronectin. This is in direct contrast to hpVICs, which tended to maintain lower-level expression of all gene targets. Interestingly, the lack of differences in gene expression on fibrin seems to be in contrast with the differences in morphology on this substrate. This result may stem from the significant difference in the expression of $\alpha_{\mathrm{v}} \beta_{3}$ and other integrins, mentioned above. The interaction of integrins with fibrin results in a significant contraction and remodeling response in vivo and in vitro in nhDFFs $[40-42,46,48]$. In one study, fibroblasts were able to almost completely replace a fibrin gel with fibrillar collagen and other ECM within nine days of culture [48]. Because of this integrin-matrix interaction, it is possible that over the long culture period the nhDFFs were able to 
produce significant amounts of new matrix, which then led to a subsequent down-regulation of matrix production and remodeling enzymes, such that the nhDFFs returned to a more "native", un-activated state, similar to hpVICs.

Figure 4. Relative expression of $\alpha$-SMA and some matrix molecules were significantly upregulated in nhDFFs (black bars) compared to hpVICs (white bars) on collagen type I (A) and fibronectin (B) at seven days in culture. Fibrin (C) elicited no significant differences in gene expression, while TCP (D) showed upregulation of only matrix-associated molecules. Data is expressed as mean $\pm \mathrm{SE}, * p \leq 0.05$, and \# denotes non-significant trends $(0.05<p<0.1)$ between cell types for the specified substrate. $n=6-8$.
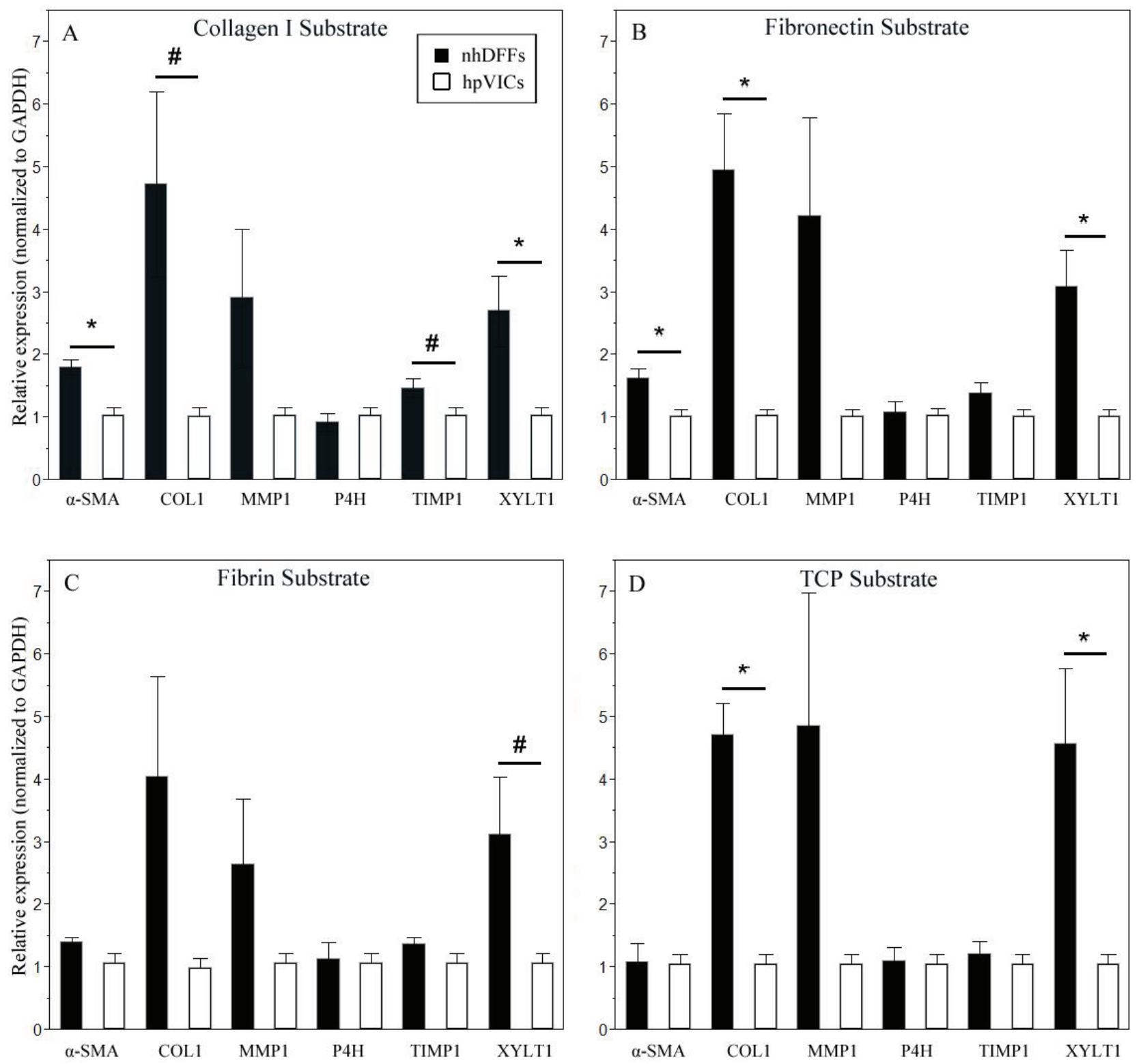

\subsection{Western Blotting}

Collagen type I and MMP1 production by both cell types was confirmed by Western blotting after 7 days in culture, as shown by representative images in Figure 5. Statistical analysis showed no significant differences in the relative protein expression between nhDFFs and hpVICs, although both 
targets were found to be significantly different when analyzing the gene expression profile. We were also able to determine that the ratio of the collagen $I \alpha 1$ and $\alpha 2$ subunits was close to 2 for all substrates (average 2.4 overall, $n=24$ ), confirming that there were no clear abnormalities in collagen production. TIMP1 was undetectable in any sample, likely due to low concentration. A lack of differences in protein expression may indicate that the cells have reached a steady-state in their protein expression that is similar for both, but that the underlying gene expression, or the propensity for the cells to take on certain characteristics when activated, seems to remain significantly different.

Figure 5. Collagen type I and MMP1 protein expression at day seven for nhDFFs (black bars) and hpVICs (white bars) was not significantly different on any culture substrate $(n=3)$. Samples were normalized to nhDFFs cultured on TCP and to a $\beta$-tubulin loading control. Error bars represent standard error. Representative Western blot images are shown for each target protein. Lanes are indicated as substrate-cell type, with substrates collagen type I (C1), fibronectin (FN), fibrin (FB), and TCP (TC), and cell types nhDFF (F) and hpVICs (V).

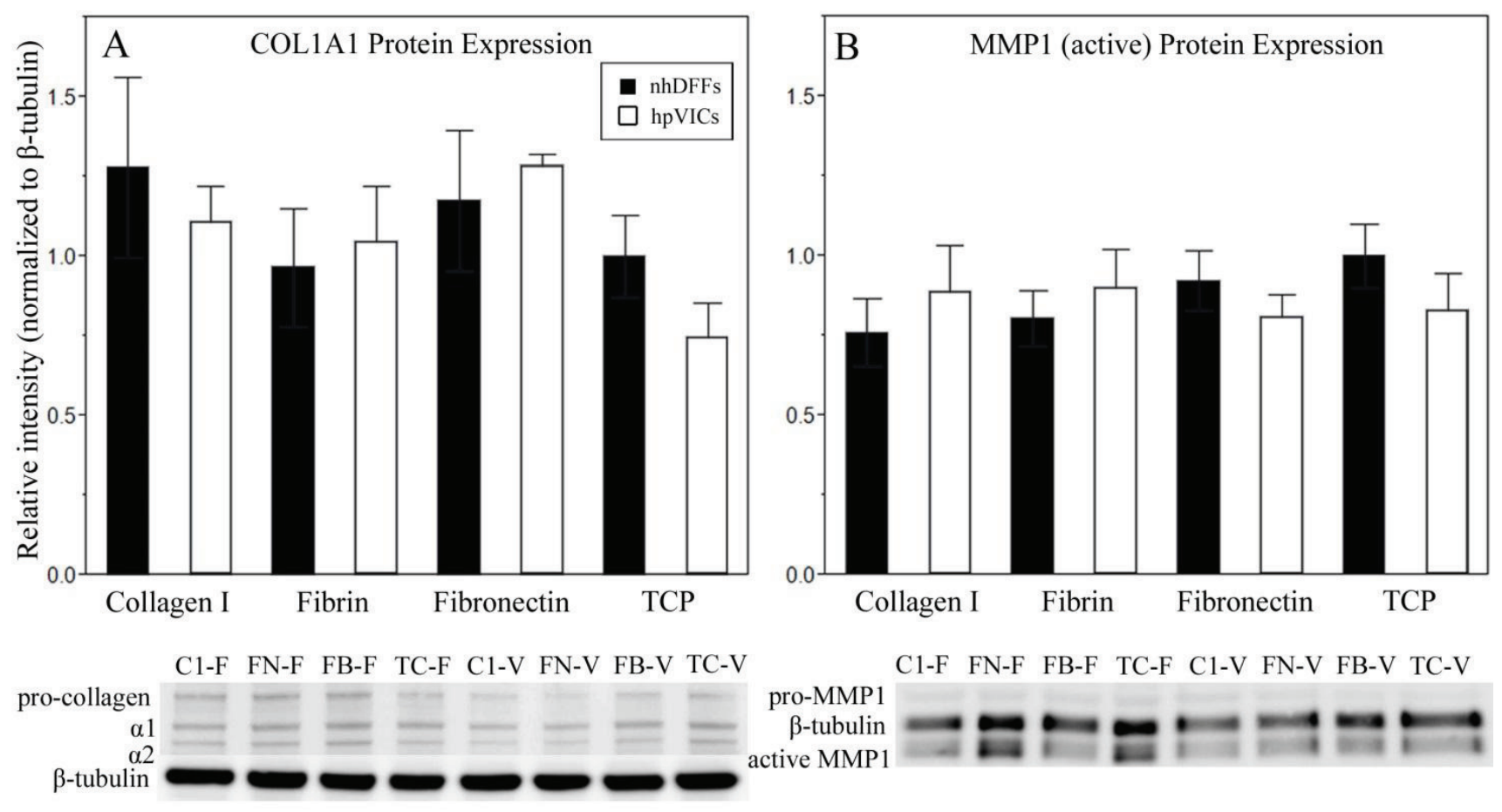

\subsection{Study Limitations}

For this study, we were unable to obtain valves from healthy donors, due to the rarity of such samples from the pediatric population. hVICs used for the study were isolated from valves that were removed due to congenital deformation. While many congenital heart defects have been linked to genetic defects in the cardiac development pathways, such as GATA4 and Tbx5, there is significant variability in the final phenotype of these conditions that suggests additional environmental influences may also be important [49]. Thus, removing these cells from a diseased condition may still result in behavior that is somewhat indicative of the native hpVIC from healthy tissue. In fact, in this work, we show that hVICs have limited and heterogeneous expression of $\alpha$-SMA and appear to be relatively slow-growing, both of which are traits similar to adult qVICs. Additionally, when cultured on our substrates, these 
cells do not seem to exhibit pathologic matrix remodeling or cell growth. Future work should be done to further investigate the proteome of these cells, and form a direct comparison to cells isolated from valves obtained at autopsy, which may help elucidate the source of congenital defects in valves. In addition, in this study, we cultured nhDFFs and hpVICs for at least a week to attempt to capture the "steady-state" behavior of these cells at long time points; however, we also showed that over this period nhDFFs and hpVICs reach significantly different levels of confluence. Cell density can be an important factor in modulating gene and protein expression, thus, future experiments should be done to isolate this as a potential factor in the gene and protein expression profiles of these cells.

\section{Conclusions}

In this work, we performed a direct comparison of dermal fibroblasts and pediatric VICs cultured on multiple matrix proteins to elucidate the similarities and differences between these two cell types in terms of functions important to valve maintenance, and the effect of matrix type on these characteristics. Of the substrates studied, fibrin elicited the most comparable behavior between nhDFFs and hpVICs, except in terms of cell growth. However, in light of previous studies, fibrin should still be considered with caution as a TEHV matrix material, as it elicits a strong wound-healing response in fibroblasts through integrin binding, and may result in too-rapid remodeling of the TEHV matrix. Culture on collagen type I, by contrast, attenuated the proliferation of fibroblasts, such that the growth of each cell type was not significantly different after the culture period. Although nhDFFs maintained significantly different levels of gene expression than hpVICs, it seemed that the steady-state matrix production was not significantly different between the two. Thus, collagen type I should be considered as a matrix component in TEHVs, especially if autologous fibroblasts are used as a VIC surrogate, since this molecule is able to control the proliferative and matrix remodeling rate of fibroblasts and encourage steady-state behavior that is more characteristic of pediatric VICs.

\section{Acknowledgments}

This work was supported by funding from the March of Dimes Research Grant 1-FY08-409. The authors wish to acknowledge the Moake Lab at Rice University for their assistance with PCR and Western blotting and Melissa McHale for her help in editing this paper. In addition, the authors would like to thank the members of the Grande-Allen Lab for conversations and advice that advanced the progress of this work.

\section{Author Contributions}

Monica Fahrenholtz contributed the majority of this work, including data collection, analysis, and manuscript preparation. Wendy Liu also contributed significantly to data collection and assisted in manuscript preparation. Lalita Wadhwa and Debra Kearney assisted in sample collection and processing in accordance with the IRB protocol. Charles Fraser and Jane Grande-Allen provided significant guidance in the conception and development of this work. 


\section{Conflicts of Interest}

The authors declare no conflict of interest.

\section{References}

1. Hoffman, J.; Kaplan, S. The incidence of congenital heart disease. J. Am. Coll. Cardiol. 2002, 39, 1890-1900.

2. Yang, Q.; Chen, H.; Correa, A.; Devine, O.; Mathews, T.J.; Honein, M.A. Racial differences in infant mortality attributable to birth defects in the United States, 1989-2002. Birth Defects Res. A Clin. Mol. Teratol. 2006, 76, 706-713.

3. Schoen, F.; Levy, R. Tissue heart valves: Current challenges and future research perspectives. J. Biomed. Mater. Res. 1999, 47, 439-465.

4. Schoen, F. Future directions in tissue heart valves: Impact of recent insights from biology and pathology. J. Heart Valve Dis. 1999, 8, 350-358.

5. Cebotari, S.; Lichtenberg, A.; Tudorache, I.; Hilfiker, A.; Mertsching, H.; Leyh, R.; Breymann, T.; Kallenbach, K.; Maniuc, L.; Batrinac, A.; et al. Clinical application of tissue engineered human heart valves using autologous progenitor cells. Circulation 2006, 114, I132-I137.

6. Allen, J.; Khan, S.; Lapidos, K.A.; Ameer, G.A. Toward Engineering a Human Neoendothelium with Circulating Progenitor Cells. Stem Cells 2010, 28, 318-328.

7. Cines, D.B.; Pollak, E.S.; Buck, C.A.; Loscalzo, J.; Zimmerman, G.A.; McEver, R.P.; Pober, J.S.; Wick, T.M.; Konkle, B.A.; Schwartz, B.S.; et al. Endothelial cells in physiology and in the pathophysiology of vascular disorders. Blood 1998, 91, 3527-3561.

8. De Mel, A.; Jell, G.; Stevens, M.M.; Seifalian, A.M. Biofunctionalization of biomaterials for accelerated in situ endothelialization: A review. Biomacromolecules 2008, 9, 2969-2979.

9. Mulholland, D.L.; Gotlieb, A.I. Cardiac Valve Interstitial Cells: Regulator of Valve Structure and Function. Cardiovasc. Pathol. 1997, 6, 167-174.

10. Taylor, P.M.; Batten, P.; Brand, N.J.; Thomas, P.S.; Yacoub, M.H. The cardiac valve interstitial cell. Int. J. Biochem. Cell Biol. 2003, 35, 113-118.

11. Rabkin-Aikawa, E.; Farber, M.; Aikawa, M.; Schoen, F.J. Dynamic and reversible changes of interstitial cell phenotype during remodeling of cardiac valves. J. Heart Valve Dis. 2004, 13, 841-847.

12. Liu, A.C.; Joag, V.R.; Gotlieb, A.I. The Emerging Role of Valve Interstitial Cell Phenotypes in Regulating Heart Valve Pathobiology. Am. J. Pathol. 2007, 171, 1407-1418.

13. Aikawa, E.; Whittaker, P.; Farber, M.; Mendelson, K.; Padera, R.F.; Aikawa, M.; Schoen, F.J. Human semilunar cardiac valve remodeling by activated cells from fetus to adult: Implications for postnatal adaptation, pathology, and tissue engineering. Circulation 2006, 113, 1344-1352.

14. Riem Vis, P.W.; Kluin, J.; Sluijter, J.P.G.; van Herwerden, L.A.; Bouten, C.V.C. Environmental regulation of valvulogenesis: Implications for tissue engineering. Eur. J. Cardio-Thoracic Surg. 2011, 39, 8-17.

15. Hinton, R.B.; Lincoln, J.; Deutsch, G.H.; Osinska, H.; Manning, P.B.; Benson, D.W.; Yutzey, K.E. Extracellular matrix remodeling and organization in developing and diseased aortic valves. Circ. Res. 2006, 98, 1431-1438. 
16. Appleton, A.J.E.; Appleton, C.T.G.; Boughner, D.R.; Rogers, K.A. Vascular Smooth Muscle Cells as a Valvular Interstitial Cell Surrogate in Heart Valve Tissue Engineering. Tissue Eng. A 2009, 15, 3889-3897.

17. Knight, R.L.; Booth, C.; Wilcox, H.E.H.; Fisher, J.; Ingham, E. Tissue engineering of cardiac valves: Re-seeding of acellular porcine aortic valve matrices with human mesenchymal progenitor cells. J. Heart Valve Dis. 2005, 14, 806-813.

18. Williams, C.; Johnson, S.L.; Robinson, P.S.; Tranquillo, R.T. Cell sourcing and culture conditions for fibrin-based valve constructs. Tissue Eng. 2006, 12, 1489-1502.

19. Taylor, P.M.; Allen, S.P.; Yacoub, M.H. Phenotypic and functional characterization of interstitial cells from human heart valves, pericardium and skin. J. Heart Valve Dis. 2000, 9, 150-158.

20. Zund, G.; Breuer, C.K.; Shinoka, T.; Ma, P.X.; Langer, R.; Mayer, J.E. The in vitro construction of a tissue engineered bioprosthetic heart valve. Eur. J. Cardio-Thoracic Surg. 1997, 11, 493-497.

21. Hoffman-Kim, D.; Maish, M.S.; Krueger, P.M.; Lukoff, H.; Bert, A.; Hong, T.; Hopkins, R.A. Comparison of three myofibroblast cell sources for the tissue engineering of cardiac valves. Tissue Eng. 2005, 11, 288-301.

22. Robinson, P.S.; Johnson, S.L.; Evans, M.C.; Barocas, V.H.; Tranquillo, R.T. Functional tissue-engineered valves from cell-remodeled fibrin with commissural alignment of cell-produced collagen. Tissue Eng. A 2008, 14, 83-95.

23. Narine, K.; DeWever, O.; Cathenis, K.; Mareel, M.; van Belleghem, Y.; van Nooten, G. Transforming Growth Factor-b-Induced Transition of Fibroblasts: A Model for Myofibroblast Procurement in Tissue Valve Engineering. J. Heart Valve Dis. 2004, 13, 281-289.

24. Narine, K.; de Wever, O.; van Valckenborgh, D.; Francois, K.; Bracke, M.; Desmet, S.; Mareel, M.; van Nooten, G. Growth Factor Modulation of Fibroblast Proliferation, Differentiation, and Invasion: Implications for Tissue Valve Engineering. Tissue Eng. 2006, 12, 2707-2716.

25. Zeltinger, J.; Landeen, L.K.; Alexander, H.G.; Kidd, I.D.; Sibanda, B. Development and characterization of tissue-engineered aortic valves. Tissue Eng. 2001, 7, 9-22.

26. Brody, S.; Pandit, A. Approaches to Heart Valve Tissue Engineering Scaffold Design. J. Biomed. Mater. Res. B Appl. Biomater. 2007, 83, 16-43.

27. Cushing, M.C.; Liao, J.-T.; Jaeggli, M.P.; Anseth, K.S. Material-based regulation of the myofibroblast phenotype. Biomaterials 2007, 28, 3378-3387.

28. Lovekamp, J.J.; Simionescu, D.T.; Mercuri, J.J.; Zubiate, B.; Sacks, M.S.; Vyavahare, N.R. Stability and function of glycosaminoglycans in porcine bioprosthetic heart valves. Biomaterials 2006, 27, 1507-1518.

29. Tseng, H.; Grande-Allen, K.J. Microstructure of the Aortic Valve: Implications for Valvular Mechanics and Function. In Advances in Cardiovascular Research, Volume 1; Schmitt, L., Konig, T., Eds.; Nova Science Publishers: Hauppauge, NY, USA, 2009; Volume 1, pp. 1-18.

30. Latif, N.; Sarathchandra, P.; Taylor, P.M.; Antoniw, J.; Yacoub, M. H. Localization and pattern of expression of extracellular matrix components in human heart valves. J. Heart Valve Dis. 2005, $14,218-227$. 
31. Flanagan, T.C.; Sachweh, J.S.; Frese, J.; Schnöring, H.; Gronloh, N.; Koch, S.; Tolba, R.H.; Schmitz-Rode, T.; Jockenhoevel, S. In vivo remodeling and structural characterization of fibrin-based tissue-engineered heart valves in the adult sheep model. Tissue Eng. A 2009, 15, 2965-2976.

32. Mol, A.; van Lieshout, M.I.; Dam-de Veen, C.G.; Neuenschwander, S.; Hoerstrup, S.P.; Baaijens, F.P.T.; Bouten, C.V.C. Fibrin as a cell carrier in cardiovascular tissue engineering applications. Biomaterials 2005, 26, 3113-3121.

33. Fondard, O.; Detaint, D.; Iung, B.; Choqueux, C.; Adle-Biassette, H.; Jarraya, M.; Hvass, U.; Couetil, J.-P.; Henin, D.; Michel, J.-B.; et al. Extracellular matrix remodelling in human aortic valve disease: The role of matrix metalloproteinases and their tissue inhibitors. Eur. Heart J. 2005, 26, 1333-1341.

34. Müller, S.; Disse, J.; Schöttler, M.; Schön, S.; Prante, C.; Brinkmann, T.; Kuhn, J.; Kleesiek, K.; Götting, C. Human xylosyltransferase I and N-terminal truncated forms: Functional characterization of the core enzyme. Biochem. J. 2006, 394, 163-171.

35. Gu, X.; Masters, K.S. Regulation of valvular interstitial cell calcification by adhesive peptide sequences. J. Biomed. Mater. Res. A 2010, 93, 1620-1630.

36. Stephens, E.H.; Carroll, J.L.; Grande-Allen, K.J. The use of collagenase III for the isolation of porcine aortic valvular interstitial cells: Rationale and optimization. J. Heart Valve Dis. 2007, 16, $175-183$.

37. Blevins, T.L.; Carroll, J.L.; Raza, A.M.; Grande-Allen, K.J. Phenotypic characterization of isolated valvular interstitial cell subpopulations. J. Heart Valve Dis. 2006, 15, 815-822.

38. Masur, S.K.; Dewal, H.S.; Dinh, T.T.; Erenburg, I.; Petridou, S. Myofibroblasts differentiate from fibroblasts when plated at low density. Proc. Natl. Acad. Sci. USA 1996, 93, 4219-4223.

39. Pfaffl, M.W. A new mathematical model for relative quantification in real-time RT-PCR. Nucleic Acids Res. 2001, 29, e45.

40. Katagiri, Y.; Hiroyama, T.; Akamatsu, N.; Suzuki, H.; Yamazaki, H.; Tanoue, K. Involvement of alpha(v)beta(3) Integrin in Mediating Fibrin Gel Retraction. J. Biol. Chem. 1995, 270, 1785-1790.

41. Gailit, J.; Clarke, C.; Newman, D.; Tonnesen, M.G.; Mosesson, M.W.; Clark, R.A. Human fibroblasts bind directly to fibrinogen at RGD sites through integrin alpha(v)beta3. Exp. Cell Res. 1997, 232, 118-126.

42. Greiling, D.; Clark, R.A. Fibronectin provides a conduit for fibroblast transmigration from collagenous stroma into fibrin clot provisional matrix. J. Cell Sci. 1997, 110, 861-870.

43. Clark, R.A.F.; Lin, F.; Greiling, D.; An, J.; Couchman, J.R. Fibroblast invasive migration into fibronectin/fibrin gels requires a previously uncharacterized dermatan sulfate-CD44 proteoglycan. J. Investig. Dermatol. 2004, 122, 266-277.

44. Fisher, C.I.; Chen, J.; Merryman, W.D. Calcific nodule morphogenesis by heart valve interstitial cells is strain dependent. Biomech. Model. Mechanobiol. 2013, 12, 5-17.

45. Wirrig, E.E.; Hinton, R.B.; Yutzey, K.E. Differential expression of cartilage and bone-related proteins in pediatric and adult diseased aortic valves. J. Mol. Cell. Cardiol. 2011, 50, 561-569.

46. Clark, R.A.F.; Nielsen, L.D.; Welch, M.P.; McPherson, J.M. Collagen matrices attenuate the collagen-synthetic response of cultured fibroblasts to TGF-(b). J. Cell Sci. 1995, 108, 1251-1261. 
47. Ku, C.-H.; Johnson, P.H.; Batten, P.; Sarathchandra, P.; Chambers, R.C.; Taylor, P.M.; Yacoub, M.H.; Chester, A.H. Collagen synthesis by mesenchymal stem cells and aortic valve interstitial cells in response to mechanical stretch. Cardiovasc. Res. 2006, 71, 548-556.

48. Tuan, T.L.; Song, A.; Chang, S.; Younai, S.; Nimni, M.E. In vitro fibroplasia: Matrix contraction, cell growth, and collagen production of fibroblasts cultured in fibrin gels. Exp. Cell Res. 1996, 223, 127-134.

49. Olson, E.N. Gene regulatory networks in the evolution and development of the heart. Science 2006, 313, 1922-1927.

\section{Supplementary Material}

Figure S1. Immunofluorescent staining of prolyl-4-hydroxylase in nhDFFs and hpVICs shows homogenous, low-level expression regardless of cell type or culture substrate.

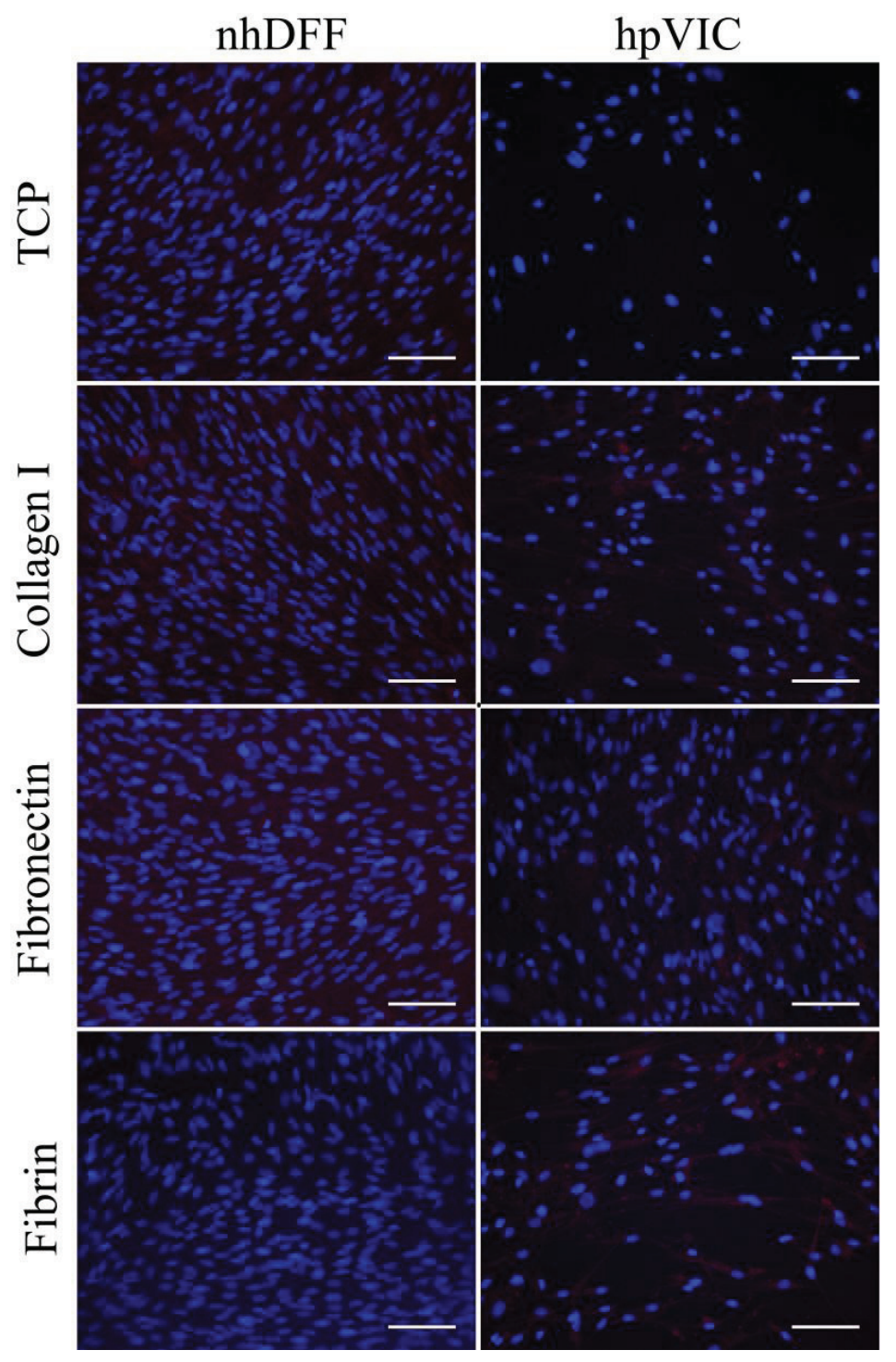

(C) 2014 by the authors; licensee MDPI, Basel, Switzerland. This article is an open access article distributed under the terms and conditions of the Creative Commons Attribution license (http://creativecommons.org/licenses/by/3.0/). 